Research Article

\title{
Decoupling Attitude Control of a Hypersonic Glide Vehicle Based on a Nonlinear Extended State Observer
}

\author{
Jian Chen $\mathbb{D}^{1,2}$ Nannan Du $\mathbb{D}^{1},{ }^{1}$ and Yu Han $\mathbb{D}^{3,4}$ \\ ${ }^{1}$ College of Engineering, China Agricultural University, Beijing 100083, China \\ ${ }^{2}$ Synergistic Innovation Center of Jiangsu Modern Agricultural Equipment and Technology, Jiangsu University, \\ Zhenjiang 212013, China \\ ${ }^{3}$ College of Water Resources \& Civil Engineering, China Agricultural University, Beijing 100083, China \\ ${ }^{4}$ State Key Laboratory of Information Engineering in Surveying, Mapping and Remote Sensing, Wuhan University, \\ Wuhan 430079, China
}

Correspondence should be addressed to Yu Han; yhan@cau.edu.cn

Received 16 July 2019; Revised 14 December 2019; Accepted 2 January 2020; Published 7 February 2020

Academic Editor: Rosario Pecora

Copyright (c) 2020 Jian Chen et al. This is an open access article distributed under the Creative Commons Attribution License, which permits unrestricted use, distribution, and reproduction in any medium, provided the original work is properly cited.

\begin{abstract}
Aiming at solving the attitude control problem of a hypersonic glide vehicle, this paper proposes a decoupling control method based on a nonlinear extended state observer (NESO). According to the decentralized robust control theory of Tornambè, the coupling terms and the uncertainties are regarded as generalized uncertainties, and the NESO-based estimation and compensation signals are added to the closed-loop control law. The theoretical deduction proves that the proposed method can ensure that the tracking error of the closed-loop system is uniformly bounded. The simulation is carried out on the hypersonic glide vehicle model and compared with the traditional subchannel feedback control method. The simulation results show that the designed decoupling control method has superior control performances, and the influence of channel-coupling and uncertainty is compensated to a great extent.
\end{abstract}

\section{Introduction}

Owing to its great space application value, the hypersonic vehicle has attracted attention all over the world. This kind of aircraft is extremely fast. Besides, a long flight in the near space, and the complexity of the fight trajectory, results in difficulty in controlling it. At present, a feasible scheme is to use a hypersonic glide vehicle with a "boost+glide" motion (as shown in Figure 1). It is a kind of plane-symmetry hypersonic vehicle with lifting-body configuration. This vehicle reentries at hypersonic speed and flies in the near space $(20-100 \mathrm{~km})$ at most of the time, of which the flight attitude is between that of the aircraft and spacecraft. Such vehicle usually adopts a large lift-to-drag ratio wave-rider configuration, and its flying Mach number is greater than 5. As shown in Figure 2, it can be sent to a predetermined height by a launch vehicle, or released by a space-based platform. After entering the atmosphere, the lift is generated by its own spe- cial aerodynamic configuration to skip glide, and it is able to fly thousands or even tens of thousands of kilometers by the "boost+glide" motion [1]. A typical representative of the hypersonic glide vehicle is the common aero vehicle (CAV) in the Falcon program jointly launched by the United States Department of Defense and the United States Air Force in 2003 [2]. The Apollo command module is basically a single-skip reentry, as is the Chinese Chang'e 5-T1. For novel vehicles like the Orion spacecraft, complicated multiskip reentry is proposed.

The special flight motion mode of the hypersonic glide vehicle puts forward a very high requirement for the design of the control system. Long-range hypersonic glide vehicles need to have some lateral maneuverability, and if they adopt side-to-turn (STT) control mode, there exist certain difficulties. When the aircraft adopts STT control mode to achieve lateral maneuvering, it needs to move sideways with a certain sideslip angle. Meanwhile, the heating and aerodynamic 


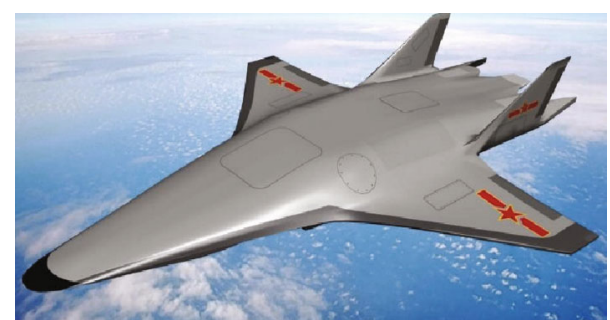

FIGURE 1: Sketch map of a hypersonic glide vehicle with the "boost +glide" motion.

performances of the aircraft vary greatly, which bring difficulties to the aircraft's overall design. The larger rudder deflection to match the turn brings greater difficulties to the aircraft's structural design and thermal protection design. If the bank-to-turn (BTT) control mode is adopted, the sideslip angle can be controlled at a small value, and the complexity to design the control surface and the thermal protection system is reduced, such that the aerodynamic efficiency is improved. Therefore, during the flight, the aircraft maneuvers laterally in the way of bank-to-turn (BTT). In order to ensure gliding in the near space, such aircraft needs to maintain a large angle of attack to provide adequate lift, while the sideslip angle should be kept as zero as possible to avoid the impact of local heating effect on the body. Moreover, in order to obtain a high lift-to-drag ratio to increase the flight range, the hypersonic glide vehicle is generally designed as a planesymmetric type of lift body, which enhances the coupling between the channels. In addition, the reentry flight speed, flight altitude, and flight range vary greatly. The flight parameters are strongly coupled and change with time. To sum up, the couplings induced by the above factors between the three channels of the aircraft are very serious, and the controlled plant-the aircraft model, is a multivariable, strongly coupled time-varying system. Especially, the coupling between the lateral channels will result in a large side angle of the aircraft, which is a serious threat to flight safety $[3,4]$. In order to guarantee the accurate tracking of the guidance command, the flight control system must be able to overcome the adverse effects of the channel coupling. Therefore, it is necessary to study the decoupling control method of MultipleInput Multiple-Output (MIMO) systems such as hypersonic glide vehicles.

In engineering, the traditional gain scheduling method is usually used to design the flight control system, but it requires a lot of storage and calculation, and it is difficult to solve the influence of the coupling effect. In [5], the decoupling control method of the aircraft is designed according to the variable structure control theory, but the highfrequency chattering caused by the frequent switching of the controller will threaten the flight safety of the aircraft. In $[6,7]$, the decoupling controller is designed using the nonlinear dynamic inverse method, but the robustness of the system cannot be guaranteed in the case that the model cannot be accurately known. In $[8,9]$, the robust decoupling control method for the linear model of the aircraft is given and the results are better, but it is not suitable for nonlinear systems such as hypersonic glide vehicles.
In [10-13], Tornambè extends the decentralized control theory from the linear Single-Input Single-Output (SISO) system [10] and the linear MIMO system [11] to a class of nonlinear MIMO system [12, 13], which can realize the independent design of the subchannel controller in the case of mastering partial nonlinear system dynamics. The channel coupling dynamic is obtained by the observer method and added as a compensation signal to the subchannel controller. Based on the decentralized robust control structure to a class of nonlinear MIMO system of which the stability is guaranteed $[12,13]$, the motivation of this paper is to estimate the channel coupling dynamic by using a nonlinear extended states observer (NESO), and finally achieve independent three channel controller design of a hypersonic glide vehicle.

The rest part of this paper is organized as follows: a decoupling control algorithm based on decentralized control theory of Tornambè and NESO is proposed in Section 2. A nonlinear MIMO model and a decoupling attitude controller of the hypersonic glide vehicle are presented in Section 3. Attitude control simulations are verified on the hypersonic glide vehicle model in Section 4, and Section 5 concludes this work.

\section{Decoupling Control Based on NESO}

2.1. MIMO System Decentralized Control. It can be shown that the decentralized control law proposed by Tornambè, which are separately designed to guarantee the stability of the system, can be locally applied to the class of square multi-input multi-output nonlinear systems described in state space form by equations of the following type $[12,13]$ :

$$
\begin{aligned}
\dot{x} & =f(x)+\sum_{i=1}^{m} g_{i}(x) u_{i}, \quad x \in \mathrm{R}^{n}, u \in \mathrm{R}^{m}, \\
y_{1} & =h_{1}(x), \\
& \ldots \quad y \in \mathrm{R}^{m}, \\
y_{m} & =h_{m}(x),
\end{aligned}
$$

where $f, g_{i}$, and $h_{i}, i=1, \cdots, m$, are differentiable functions of their arguments: $f, g_{i} \in C^{p}\left(\mathrm{R}^{n}, \mathrm{R}^{n}\right)$, and $h_{i} \in C^{p}\left(\mathrm{R}^{n}, \mathrm{R}\right)$, with $p>0$ being a suitable integer, $n$ is the dimension of the state vector, $y=\left(y_{1}, \cdots, y_{m}\right)^{\mathrm{T}}, u=\left(u_{1}, \cdots, u_{m}\right)^{\mathrm{T}}, G=\left(g_{1}, \cdots, g_{m}\right)$, and $h=\left(h_{1}, \cdots, h_{m}\right)^{\mathrm{T}}$. And Tornambè applied this separation principle procedure to a high-nonlinear robotic manipulator model $[12,13]$.

Thus, in this paper, a class of nonlinear MIMO system is considered as follows:

$$
\left\{\begin{array}{l}
\dot{x}=f(x)+\sum_{i=1}^{n} g_{i}(x) u_{i}=f(x)+g(x) u \\
y=x
\end{array}\right.
$$

Among them, $x \in R^{n}$ is the state variable of the system, $u \in R^{n}$ is the control input of the system, $y=x \in R^{n}$ is the output of the system, and $f(x)$ and $g(x)$ are the continuously 


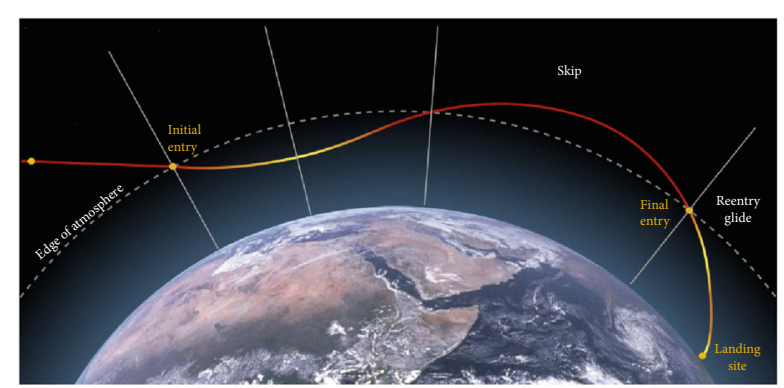

FIGURE 2: Sketch map of the "boost+glide" motion.

differentiable nonlinear functions. Due to the uncertainty and the influence of the unmodeled dynamics, the values of $f(x)$ and $g(x)$ cannot be accurately obtained. For a hypersonic glide vehicle, the elements of $f(x)$ contain aerodynamic force and moment parameters, and the elements of $g(x)$ contain the rudder parameters, which are high uncertainties.

The control objective is to make the real output of the system accurately track the expected value on the basis of ensuring the stability of the system:

$$
x=x_{\mathrm{des}},
$$

where $x_{\text {des }}$ is the desired output of system (2).

Define the output tracking error:

$$
\varepsilon=x-x_{\mathrm{des}} .
$$

Based on the decentralized control idea of Tornambè in $[12,13]$, the $i$ th equation in the differential equations of system (2) is rewritten as follows:

$$
\dot{x}_{i}=u_{i}+f_{i}(x)+g_{i}(x) u-u_{i}, \quad i=1, \cdots n .
$$

Define the generalized uncertainties of the system $[12,13]$ :

$$
\begin{aligned}
\Delta_{i} & =f_{i}(x)+g_{i}(x) u-u_{i}, \quad i=1, \cdots n, \\
\Delta & =\left[\Delta_{1}, \cdots, \Delta_{i}, \cdots \Delta_{n}\right]^{\mathrm{T}} .
\end{aligned}
$$

Then, the MIMO system (2) can be expressed as follows:

$$
\left\{\begin{array}{l}
\dot{x}_{i}=u_{i}+\Delta_{i}, \\
y=x
\end{array} \quad i=1,2, \cdots, n,\right.
$$

In equation (7), the generalized uncertainty $\Delta_{i}$ not only contains the parameter uncertainty and unmodeled dynamics of the system but also contains the dynamic effect caused by the coupling terms.

After obtaining the estimated value $\widehat{\Delta}_{i}$ of the $\Delta_{i}$ with the observer approach in the later Subsection 2.2, the control law of system (2) can be given as follows:

$$
u_{i}=-k_{i} \varepsilon_{i}-\widehat{\Delta}_{i}+\dot{x}_{i, \mathrm{des}}, \quad i=1, \cdots n,
$$

where $k_{i}$ is a feedback gain and $\varepsilon_{i}$ represents the tracking error between the desired output $x_{i \text {,des }}$ and the state $x_{i}$.

By utilizing the control law given by equation (8), system (2) can be converted into $\dot{\varepsilon}_{i}+k_{i} \varepsilon_{i}=0$. If $k_{i}$ is determined to be a positive value, the characteristic roots (eigenvalues) of system (2) are placed in the open left half-plane and hence the system is stable. The dynamical performances of system (2) can be reached by tuning the value of $k_{i}$.

It can be seen from (8) that the control law can be solved without knowing the exact value of $f(x)$ and $g(x)$, which is robust to parameter uncertainties and unmodeled dynamics. In addition, the system state and system input is a one-to-one correspondence, which shows that the designed control law is decentralized and decoupling.

2.2. Nonlinear Extended State Observer (NESO). The nonlinear extended state observer (NESO) can be used to estimate the uncertainties of a nonlinear system. It can be seen from equation (2) that the $n$th equation in the MIMO system represents the $n$th channel of the system which can independently design the controller. Correspondingly, the $n$th NESO needs to be designed to give the estimated values of the generalized uncertainty.

Using the second-order NESO [14], the $i$ th state equation's generalized uncertainty is extended to a new state, and the following observer equation can be obtained:

$$
\left\{\begin{array}{l}
e_{1, i}=z_{1, i}-x_{i}, \\
e_{2, i}=z_{2, i}-\Delta_{i}, \\
\dot{z}_{1, i}=z_{2, i}-\beta_{1, i} e_{1 . i}+u_{i}, \\
\dot{z}_{2, i}=-\beta_{2, i} f a l\left(e_{1, i}, a, \delta\right),
\end{array}\right.
$$

where

$$
f a l\left(e_{1, i}, a, \delta\right)=\left\{\begin{array}{l}
\left|e_{1, i}\right|^{a} \operatorname{sign}\left(e_{1, i}\right), \quad\left|e_{1, i}\right|>\delta \\
\frac{e_{1, i}}{\delta^{1-a}}, \quad\left|e_{1, i}\right| \leq \delta
\end{array}\right.
$$

where $\beta_{1, i}, \beta_{2, i}, a$, and $\delta$ are the NESO observer parameters to be determined. The extended state $z_{2, i}$ is an estimate of $\Delta_{i}$, and $e_{2, i}$ is the estimation error between $z_{2, i}$ and $\Delta_{i}$.

Remark 1. There is a sign function in the fal function (10) of NESO. The sign function utilizes the switching and the sliding mode strategies to cope with effects due to system uncertainties. The conventional high-frequency chattering phenomenon arising from sliding mode technique might occur in the system's observer and control signals. Thus, some scholars use more complex nonlinear smooth functions to replace with the nonsmooth $\mathrm{fal}$ function to overcome the drawback. For realizing easily in engineering, in this paper, we overcome the high-frequency chattering phenomenon by tuning the parameters and gains. However, the phenomenon is hard to be eliminated completely since NESO uses the sliding mode technique to observe and compensate uncertainties. Actually, sometimes, the steady-state error of the 
controller with the NESO will be a little larger than that of the controller without NESO since the high-frequency chattering phenomenon is occurring. However, the overshoot and settling time of the controller with the NESO are both superior to those of the controller without NESO. The performances of the controller with NESO are better than those of the controller without NESO in general.

The convergence proof of NESO and the relevant parameters' tuning rules are given in Theorem 2 .

Theorem 2 [14]. For a nonlinear system (2) and a designed NESO observer (9), assume that $\left|\dot{\Delta}_{i}\right| \leq \bar{\Delta}_{i}, i=1, \cdots n$. If the undetermined parameter satisfies $0.5 \beta_{1, i}^{2}>\beta_{2, i}>\bar{\Delta}_{i}$ and $\alpha=$ $\alpha^{*}$, then $\delta$ exists, which make the following hold:

$$
\begin{aligned}
r_{0, i}=\left(\frac{\bar{\Delta}_{i}}{\beta_{2, i}}\right)^{1 / \alpha^{*}} & =\frac{2 \bar{\Delta}_{i}}{\beta_{1, i}^{2}}+\left(\frac{2 \beta_{2, i}}{\beta_{1, i}^{2}}\right)^{1 /\left(1-a^{*}\right)} \alpha^{\alpha^{*} /\left(1-\alpha^{*}\right)}\left(1-\alpha^{*}\right), \\
& \left|\left(\frac{2 \beta_{2, i} \delta^{a^{*}}}{\beta_{1, i}^{2}}\right)-\delta\right| \leq r_{0, i} .
\end{aligned}
$$

Then, the estimation error of NESO is bounded. That is,

$$
G_{0}=\left\{\left(e_{1, i}, e_{2, i}\right)\left|e_{1, i}\right| \leq r_{0, i},\left|e_{2, i}\right| \leq \beta_{1, i} r_{0, i}\right\} .
$$

2.3. Decoupling Control Based on NESO. Different from references $[12,14]$, there is a first innovation in this section. Combined with the estimation result of NESO, the decoupling control law is proposed and rewritten as follows:

$$
u_{i}=-k_{i} \varepsilon_{i}-z_{2, i}+\dot{x}_{i, \mathrm{des}}, \quad i=1, \cdots n .
$$

The estimation result of NESO is added to the control law as an estimate of the generalized uncertainty. Theorem 3 proved that the stability of the closed-loop system and the convergence of the tracking error can be guaranteed with the decoupling control law (13).

Theorem 3. For the closed-loop system composed of the MIMO system (2) and NESO (9), if the control law (13) is adopted, then the tracking error of the closed-loop system is uniformly bounded.

Proof. Given the following Lyapunov function:

$$
\begin{aligned}
& V=\frac{1}{2} \sum_{i=1}^{n} \varepsilon_{i}^{2}, \\
& \dot{V}=\sum_{i=1}^{n} \varepsilon_{i} \dot{\varepsilon}_{i}=\sum_{i=1}^{n} \varepsilon_{i}\left(\dot{x}_{i}-\dot{x}_{i, \mathrm{des}}\right)=\sum_{i=1}^{n} \varepsilon_{i}\left(u_{i}+\Delta_{i}-\dot{x}_{i, \mathrm{des}}\right) .
\end{aligned}
$$

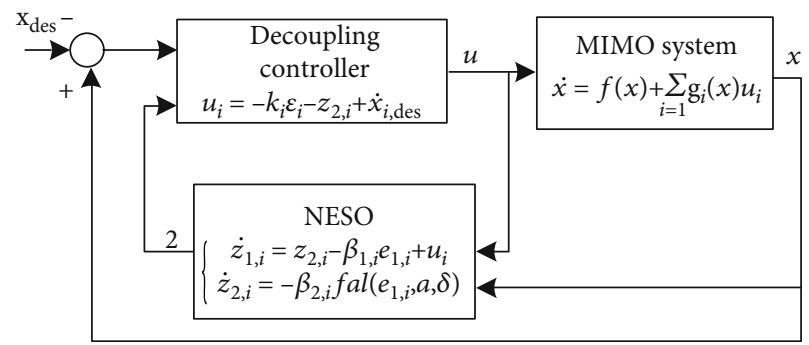

Figure 3: Block diagram of a NESO-based decoupling controller.

Substituting the expression of the control law, the following formula is available:

$$
\begin{aligned}
\dot{V} & =\sum_{i=1}^{n} \varepsilon_{i}\left(-k_{i} \varepsilon_{i}-z_{2, i}+\dot{x}_{i, \mathrm{des}}+\Delta_{i}-\dot{x}_{i, \mathrm{des}}\right) \\
& =\sum_{i=1}^{n}\left(-k_{i} \varepsilon_{i}^{2}-\varepsilon_{i} e_{2, i}\right) \leq \sum_{i=1}^{n}\left(-k_{i} \varepsilon_{i}^{2}+\left|\varepsilon_{i}\right|\left|e_{2, i}\right|\right),
\end{aligned}
$$

where the extended state $z_{2, i}$ is an estimate of the uncertainty $\Delta_{i}$ from NESO's equation (9) and $e_{2, i}$ is the estimation error between $z_{2, i}$ and $\Delta_{i}$ from NESO's equation (9). Obviously, $e_{2, i}$ has a great influence on the Lyapunov function's boundedness. Thus, Theorem 3 is proved with the Lyapunov function with NESO.

Then, according to the conclusion given by Theorem 2 (the convergence proof of the NESO), there exists

$$
\dot{V} \leq \sum_{i=1}^{n}\left(-k_{i} \varepsilon_{i}^{2}+\beta_{1, i} r_{0, i}\left|\varepsilon_{i}\right|\right) \text {. }
$$

When the following formula is established:

$$
\left|\varepsilon_{i}\right|>\frac{\beta_{1, i} r_{0, i}}{k_{i}}, \quad i=1,2, \cdots n
$$

there exists $\dot{V}<0$ that the tracking error of the closed-loop system is uniformly bounded.

Figure 3 is a control block diagram of the closed-loop system. The input signal of the decoupling controller is the MIMO system's tracking error and the NESO's estimated value for the generalized uncertainties. The control law is a subchannel designed by the decentralized control theory. The input signal of the NESO is the control input and system output of the MIMO system. It can be seen from the block diagram that the NESO-based decoupling control method combines the traditional subchannel design method and the observer method. On the basis of guaranteeing the decoupling control effect and the robustness of the system, the traditional subchannel controllers' design experiences can be used to facilitate the engineering implementation. 


\section{Decoupling Control of the Hypersonic Glide Vehicle}

3.1. Modeling of the Hypersonic Glide Vehicle. Assuming that the hypersonic glide vehicle is a plane-symmetry rigid body, ignoring the Earth's rotation and the mass change of the aircraft, the following simplified model can be derived according to theoretical mechanics principles [15].

Establish the centroid dynamics equation in the track coordinate system:

$$
\begin{aligned}
{\left[\begin{array}{c}
m \dot{V} \\
m V \dot{\theta} \\
-m V \dot{\psi}_{s} \cos \theta
\end{array}\right]=} & \mathbf{B}_{d}^{t} \mathbf{B}_{b}^{d}\left[\begin{array}{c}
Q_{x 1} \\
Q_{y 1} \\
Q_{z 1}
\end{array}\right]-\left[\begin{array}{c}
m g \sin \theta \\
m g \cos \theta \\
0
\end{array}\right]+m V^{2}\left[\begin{array}{c}
0 \\
\frac{\cos \theta}{r} \\
\left.\frac{-\cos ^{2} \theta \sin \psi_{s} \tan \phi}{r}\right]
\end{array}\right] \\
& +m \omega_{D}^{2} r \cos \phi\left[\begin{array}{c}
\sin \theta \cos \phi-\cos \theta \cos \psi_{s} \sin \phi \\
\cos \theta \cos \phi+\sin \theta \cos \psi_{s} \sin \phi \\
-\sin \psi_{s} \sin \phi
\end{array}\right]+2 m V \omega_{D}\left[\begin{array}{c}
0 \\
-\cos \phi \sin \psi_{s} \\
\cos \theta \sin \phi-\cos \phi \sin \theta \cos \psi_{s}
\end{array}\right] .
\end{aligned}
$$

The five terms on the right side of the equation are as follows: aerodynamics $\mathbf{Q}$, gravity $\mathbf{G}$, centrifugal force $\mathbf{S}_{v}$ caused by high-speed flight, centrifugal force $\mathbf{S}_{e}$ caused by earth rotation, and the projection of Coriolis inertial force $\mathbf{S}_{c}$ caused by earth rotation in the ballistic coordinate system. In the hypersonic reentry flight, the effects of centrifugal force and Coriolis inertial force caused by high-speed flight cannot be neglected compared with the external force, especially that the centrifugal force caused by high-speed flight may be very large.

The position of the aircraft is determined by three parameters: geocentric distance $r=R_{E}+H$, longitude $\lambda$, and lati- tude $\phi$. The navigation kinematics equations represented by polar coordinates are as follows:

$$
\left\{\begin{array}{l}
\dot{r}=V \sin \theta, \\
\dot{\phi}=\frac{V \cos \theta \cos \psi_{s}}{r}, \\
\dot{\lambda}=\frac{-V \cos \theta \sin \psi_{s}}{r \cos \phi} .
\end{array}\right.
$$

Establish a system of moment equations in the body coordinate system:

$$
\left\{\begin{array}{l}
\dot{\omega}_{x 1}=\frac{J_{y} \cdot M_{x 1}}{J_{x} J_{y}-J_{x y}{ }^{2}}+\frac{J_{x y} \cdot M_{y 1}}{J_{x} J_{y}-J_{x y}{ }^{2}}-\frac{J_{x y}\left(J_{x}+J_{y}-J_{z 1}\right)}{J_{x} J_{y}-J_{x y}{ }^{2}} \omega_{z 1} \omega_{x 1}+\frac{J_{y}{ }^{2}+J_{x y}{ }^{2}-J_{y} J_{z}}{J_{x} J_{y}-J_{x y}{ }^{2}} \omega_{y 1} \omega_{z 1}, \\
\dot{\omega}_{y 1}=\frac{J_{x} \cdot M_{y 1}}{J_{x} J_{y}-J_{x y}{ }^{2}}+\frac{J_{x y} \cdot M_{x 1}}{J_{x} J_{y}-J_{x y}{ }^{2}}-\frac{J_{x y}\left(J_{z}-J_{x}-J_{y}\right)}{J_{x} J_{y}-J_{x y}{ }^{2}} \omega_{y 1} \omega_{z 1}+\frac{J_{x} J_{z}-J_{x y}^{2}-J_{x}^{2}}{J_{x} J_{y}-J_{x y}{ }^{2}} \omega_{z 1} \omega_{x 1}, \\
\dot{\omega}_{z 1}=\frac{M_{z 1}}{J_{z}}-\frac{J_{y}-J_{x}}{J_{z}} \omega_{x 1} \omega_{y 1}+\frac{J_{x y}}{J_{z}}\left(\omega_{x 1}^{2}-\omega_{y 1}^{2}\right),
\end{array}\right.
$$

where $M_{x 1}, M_{y 1}, M_{z 1}$ is the projection of the combined external moment acting on the aircraft in the body coordinate system and $\omega_{x 1}, \omega_{y 1}, \omega_{z 1}$ refers to the component of the absolute angular velocity of the aircraft in the body coordinate system.

Hypothesis 1. Ignoring the influence of wind, the flight speed $\mathbf{V}$ is equal to the airspeed $\mathbf{U}$, the speed coordinate system coincides with the airflow coordinate system, the angle of attack $\alpha$ is the angle between the projection of the flight speed on the longitudinal symmetry plane of the body and the longitudinal axis of the body, the sideslip angle $\beta$ is the included angle between the flight speed and the longitudinal symmetry plane of the airframe, and the bank angle $\gamma_{s}$ is the roll angle of the aircraft around the flight speed vector.

Hypothesis 2. Ignore the effects of implicated inertial forces. This term is several orders of magnitude smaller than other forces. 
Hypothesis 3. The product of inertia is small and negligible, i.e., $J_{x y}, J_{y z}, J_{x z}=0, \mathbf{J}=\operatorname{diag}\left\{J_{x}, J_{y}, J_{z}\right\}$.
By simplifying (18) and (19), the kinematics and dynamics equations of the center of mass are as follows:

$$
\begin{aligned}
& \left\{\begin{array}{l}
\dot{r}=V \sin \theta, \\
\dot{\phi}=\frac{V \cos \theta \cos \psi_{s}}{r}, \\
\dot{\lambda}=\frac{-V \cos \theta \sin \psi_{s}}{r \cos \phi},
\end{array}\right. \\
& \left\{\begin{array}{l}
\dot{V}=-D-g \sin \theta, \\
\dot{\theta}=\frac{1}{V}\left[L \cos \gamma_{s}-Y \sin \gamma_{s}+\left(\frac{V^{2}}{r-g}\right) \cos \theta-2 V \omega_{D} \cos \phi \sin \psi_{s}\right], \\
\dot{\psi}_{s}=\frac{1}{V \cos \theta}\left[-L \sin \gamma_{s}-Y \cos \gamma_{s}+\left(\frac{V^{2} \cos ^{2} \theta \sin \psi_{s} \tan \phi}{r-2 V \omega_{D}}\right)\left(\cos \theta \sin \phi-\cos \phi \sin \theta \cos \psi_{s}\right)\right],
\end{array}\right.
\end{aligned}
$$

where $r$ is the distance of the aircraft from the center of the earth, $\phi$ is latitude, $\lambda$ is longitude, $V$ is the magnitude of flight speed, $\theta$ is the ballistic inclination angle, $\psi_{s}$ is the ballistic declination angle (the projection of the flight speed in the horizontal plane is to the left of the north direction is positive), $g$ is the gravity acceleration, $D$ is the resistance acceleration, $L$ is the lift acceleration, $Y$ is the side force acceleration, $\omega_{D}$ is the earth rotation angular velocity, and $\gamma_{s}$ is the bank angle.
According to the angular velocity transfer relationship between the coordinate systems, the following equation holds:

$$
\left(\boldsymbol{\omega}_{q-d}\right)_{q}+\left(\boldsymbol{\omega}_{d-i}\right)_{q}=\left(\boldsymbol{\omega}_{q-b}\right)_{q}+\left(\boldsymbol{\omega}_{b-i}\right)_{q}
$$

where

$$
\begin{aligned}
& \left(\boldsymbol{\omega}_{q-d}\right)_{q}=\left[\begin{array}{c}
\dot{\gamma}_{s}+\dot{\psi}_{s} \sin \theta \\
\dot{\theta} \sin \gamma_{s}+\dot{\psi}_{s} \cos \theta \cos \gamma_{s} \\
\dot{\theta} \cos \gamma_{s}-\dot{\psi}_{s} \cos \theta \sin \gamma_{s}
\end{array}\right] \\
& \left(\boldsymbol{\omega}_{d-i}\right)_{q}=\overline{\mathbf{B}}_{d}^{q}\left[\begin{array}{c}
\omega_{D} \cos \phi \\
\omega_{D} \sin \phi \\
0
\end{array}\right]+\overline{\mathbf{B}}_{d}^{q}\left[\begin{array}{c}
\dot{\lambda} \cos \phi \\
\dot{\lambda} \sin \phi \\
-\dot{\phi}
\end{array}\right] \\
& \left(\boldsymbol{\omega}_{q-b}\right)_{q}=\left[\begin{array}{c}
-\dot{\alpha} \sin \beta \\
-\dot{\beta} \\
-\dot{\alpha} \cos \beta
\end{array}\right] \\
& \left(\boldsymbol{\omega}_{b-i}\right)_{q}=\mathbf{B}_{b}^{q}\left[\begin{array}{c}
\omega_{x 1} \\
\omega_{y 1} \\
\omega_{z 1}
\end{array}\right]=\left[\begin{array}{c}
\omega_{x 1} \cos \alpha \cos \beta-\omega_{y 1} \sin \alpha \cos \beta+\omega_{z 1} \sin \beta \\
\omega_{x 1} \sin \alpha+\omega_{y 1} \cos \alpha \\
-\omega_{x 1} \cos \alpha \sin \beta+\omega_{y 1} \sin \alpha \sin \beta+\omega_{z 1} \cos \beta
\end{array}\right], \\
& \overline{\mathbf{B}}_{d}^{q}=\left[\begin{array}{ccc}
\cos \theta \cos \psi_{s} & \sin \theta & -\cos \theta \sin \psi_{s} \\
-\cos \gamma_{s} \sin \theta \cos \psi_{s}+\sin \gamma_{s} \sin \psi_{s} & \cos \gamma_{s} \cos \theta & \cos \gamma_{s} \sin \theta \sin \psi_{s}+\sin \gamma_{s} \cos \psi_{s} \\
\sin \gamma_{s} \sin \theta \cos \psi_{s}+\cos \gamma_{s} \sin \psi_{s} & -\sin \gamma_{s} \cos \theta & -\sin \gamma_{s} \sin \theta \sin \psi_{s}+\cos \gamma_{s} \cos \psi_{s}
\end{array}\right] .
\end{aligned}
$$


From (22) and (23), we can get the equation of attitude motion:

$$
\begin{aligned}
& {\left[\begin{array}{c}
\dot{\alpha} \\
\dot{\beta} \\
\dot{\gamma}_{s}
\end{array}\right]=} {\left[\begin{array}{ccc}
-\cos \alpha \tan \beta & \sin \alpha \tan \beta & 1 \\
\sin \alpha & \cos \alpha & 0 \\
\cos \alpha \sec \beta & -\sin \alpha \sec \beta & 0
\end{array}\right]\left[\begin{array}{c}
\omega_{x 1} \\
\omega_{y 1} \\
\omega_{z 1}
\end{array}\right] } \\
& \frac{g \cos \gamma_{s} \cos \theta-L}{V \cos \beta} \\
& \frac{g \sin \gamma_{s} \cos \theta+Y}{V} \\
&+\left[\begin{array}{c}
\frac{\left(g \cos \gamma_{s} \cos \theta-L\right) \tan \beta}{V}
\end{array}\right]
\end{aligned}
$$

From hypothesis 3, equation (20) can be simplified to obtain the dynamic equation of attitude rotation as follows:

$$
\left[\begin{array}{l}
\dot{\omega}_{x 1} \\
\dot{\omega}_{y 1} \\
\dot{\omega}_{z 1}
\end{array}\right]=\left[\begin{array}{ccc}
\frac{1}{J_{x}} & 0 & 0 \\
0 & \frac{1}{J_{y}} & 0 \\
0 & 0 & \frac{1}{J_{z}}
\end{array}\right]\left[\begin{array}{l}
M_{x 1} \\
M_{y 1} \\
M_{z 1}
\end{array}\right]+\left[\begin{array}{c}
c_{2} \omega_{y 1} \omega_{z 1} \\
c_{4} \omega_{x 1} \omega_{z 1} \\
c_{5} \omega_{x 1} \omega_{y 1}
\end{array}\right],
$$

where $c_{2}=\left(J_{y}-J_{z}\right) / J_{x}, c_{4}=\left(J_{z}-J_{x}\right) / J_{y}$, and $c_{5}=\left(J_{x}-J_{y}\right) / J_{z}$. $\omega_{x 1}, \omega_{y 1}, \omega_{z 1}$ is the component of the absolute angular velocity of the aircraft in the body coordinate system, $J_{x}, J_{y}, J_{z}$ is the moment of inertia, and $M_{x 1}, M_{y 1}, M_{z 1}$ is the component of the external moment acting on the aircraft in the body coordinate system.

The aircraft models (29) and (30) can be organized into the following block forms:

$$
\begin{aligned}
& \dot{\mathbf{\Omega}}=\mathbf{f}_{1}(\boldsymbol{\Omega}, \mathbf{u})+\mathbf{N}(\boldsymbol{\Omega}) \boldsymbol{\omega}, \\
& \dot{\boldsymbol{\omega}}=\mathbf{f}_{2}(\boldsymbol{\Omega}, \boldsymbol{\omega})+\mathbf{B}(\boldsymbol{\Omega}) \mathbf{u}, \\
& \mathbf{f}_{1}(\boldsymbol{\Omega}, \mathbf{u})=\left[\begin{array}{c}
\frac{g \cos \gamma_{s} \cos \theta-L}{V \cos \beta} \\
\frac{g \sin \gamma_{s} \cos \theta+Y}{V} \\
-\dot{\psi}_{s} \sin \theta-\frac{\left(g \cos \gamma_{s} \cos \theta-L\right) \tan \beta}{V}
\end{array}\right] \text {, } \\
& \mathbf{N}(\boldsymbol{\Omega})=\left[\begin{array}{ccc}
-\cos \alpha \tan \beta & \sin \alpha \tan \beta & 1 \\
\sin \alpha & \cos \alpha & 0 \\
\cos \alpha \sec \beta & -\sin \alpha \sec \beta & 0
\end{array}\right] \text {, } \\
& \mathbf{f}_{2}(\boldsymbol{\Omega}, \boldsymbol{\omega})=\mathbf{J}^{-1}\left(-\boldsymbol{\omega} \times \mathbf{J} \boldsymbol{\omega}+\mathbf{M}_{0}+\mathbf{M}_{\mathrm{damp}}+\mathbf{M}_{d}\right), \\
& \mathbf{B}(\boldsymbol{\Omega})=\mathbf{J}^{-1} \mathbf{C}_{\delta}(\boldsymbol{\Omega}),
\end{aligned}
$$

where $\boldsymbol{\Omega}=\left[\alpha, \beta, \gamma_{s}\right]^{\mathrm{T}}$ is the state vector including the bank angle, sideslip angle, and angle of attack; $\mathbf{u}=\left[\delta_{a}, \delta_{r}, \delta_{e}\right]^{\mathrm{T}}$ is the control vector including the actual rudder deviations of the differential aileron, rudder and elevator; and $\boldsymbol{\omega}=$ $\left[\omega_{x 1}, \omega_{y 1}, \omega_{z 1}\right]^{\mathrm{T}}$ is body shaft angular velocity vector. $\psi_{s}$ and $\theta$ are the trajectory declination and inclination, $g$ is the acceleration of gravity, $L$ and $Y$ are the accelerations of lift and side forces, $V$ is the speed of flight, $\mathbf{J}$ is the matrix of inertia, $\mathbf{M}_{0}$ is the moment generated by the basic body, $\mathbf{M}_{\text {damp }}$ is the damping moment, $\mathbf{M}_{d}$ is the disturbance moment, and $\mathbf{C}_{\delta}$ is the aerodynamic moment parameter matrix.

The aerodynamic force generated by the aerodynamic rudder surface is at least 1-2 orders of magnitude smaller than the aerodynamic force generated by the basic body, so the aerodynamic force generated by the rudder surface deflection can be approximately ignored. The force acting on the aircraft is determined by the flight attitude.

$$
\left\{\begin{array}{l}
L \approx L_{0}\left(M_{a}, H, \alpha, \beta\right), \\
D \approx D_{0}\left(M_{a}, H, \alpha, \beta\right), \\
Y \approx Y_{0}\left(M_{a}, H, \alpha, \beta\right) .
\end{array}\right.
$$

In addition, since the ballistic inclination of the glide section is usually very small, there is $\cos \theta \approx 1$ and $\sin \theta \approx 0$. Therefore, the nonlinear dynamics $\mathbf{f}_{1}(\boldsymbol{\Omega}, \mathbf{u})$ in (31) can be further simplified as

$$
\mathbf{f}_{1}(\boldsymbol{\Omega})=\left[\begin{array}{c}
-\frac{\left(g \cos \gamma_{s}-L_{0}\right) \tan \beta}{V} \\
\frac{g \sin \gamma_{s}+Y_{0}}{V} \\
\frac{g \cos \gamma_{s}-L_{0}}{V \cos \beta}
\end{array}\right] .
$$

Finally, the model of the hypersonic glide vehicle flight attitude control system is obtained as the following cascade affine nonlinear system:

$$
\begin{aligned}
& \dot{\boldsymbol{\Omega}}=\mathbf{f}_{1}(\boldsymbol{\Omega})+\mathbf{N}(\boldsymbol{\Omega}) \boldsymbol{\omega}, \\
& \dot{\boldsymbol{\omega}}=\mathbf{f}_{2}(\boldsymbol{\Omega}, \boldsymbol{\omega})+\mathbf{B}(\boldsymbol{\Omega}) \mathbf{u} .
\end{aligned}
$$

Because the flight state variables have significant differences in time scale, singular perturbation theory is used to decompose the vehicle state variables into different speed loops. The control law is applied to the controlled plant "hypersonic glide vehicle" by controlling the rudder surfaces. When the rudder surface changes, the vehicle's angular rate $\boldsymbol{\omega}=\left[\omega_{x 1}, \omega_{y 1}, \omega_{z 1}\right]^{\mathrm{T}}$ changes first, and then the attitude angle $\boldsymbol{\Omega}=\left[\alpha, \beta, \gamma_{s}\right]^{\mathrm{T}}$ changes. Therefore, $\boldsymbol{\omega}=\left[\omega_{x 1}, \omega_{y 1}, \omega_{z 1}\right]^{\mathrm{T}}$ is called the fast state of the system and $\boldsymbol{\Omega}=\left[\alpha, \beta, \gamma_{s}\right]^{\mathrm{T}}$ is called the slow state.

3.2. Coupling Analysis of the Hypersonic Glide Vehicle. Hypersonic glide vehicle is a complex type of MIMO system. 


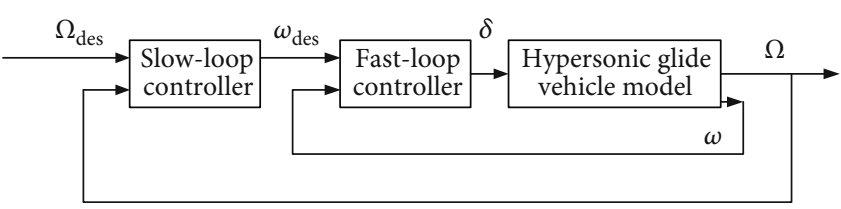

FIGURE 4: Structure of the fast-and-slow-loop flight control system.

The control input is the rudder, elevator, and aileron deflection of the yaw, pitch, and roll channel, and the output is the angle of attack, sideslip angle, and bank angle. Using the traditional subchannel control design method, the pitch channel state variables are the angle of attack and the pitch rate, and the yaw channel state variables are the sideslip angle and the yaw rate. The roll channel is the bank angle and the roll rate. Besides, when designing the channel control law, the coupling between the channels is not taken into account by the traditional subchannel control design method.

It can be seen from equations (37) to (39) that the coupling between the channels is reflected in the following three aspects [16]:

(1) Kinematic coupling terms

(2) Coupling terms caused by the product of inertia between the roll and yaw channels

(3) Coupling items caused by aerodynamic forces between the channels

Due to the special flight mode of the hypersonic glide vehicle, that is, the high-angle-attack flight and large bankangle turnover, the value of the above coupling items will become very large, resulting in that the coupling of the aircraft between the three channels is very serious, which is beyond the range of control capability of traditional subchannel method. Considering the strict requirements of the aircraft for guidance-command tracking and attitude stabilization, it is necessary to design the attitude decoupling controller to ensure the success of the flight mission.

3.3. Attitude Decoupling Control of the Hypersonic Glide Vehicle. The purpose of the hypersonic glide vehicle' attitude decoupling control is to track the airflow angle command given by the guidance system under the premise of ensuring the stability of the attitude. That is,

$$
\Omega_{\mathrm{des}}=\left[\alpha_{\mathrm{des}}, \beta_{\mathrm{des}}, \gamma_{\text {sdes }}\right]^{\mathrm{T}} .
$$

Remark 4. Time-scale separation principle. The influence of the steering force generated by the deflection of the control surface of the hypersonic glide vehicle is much smaller than that of the steering torque, so the attitude kinetic equation of the hypersonic glide vehicle is divided into ("fast and slow") two loops for the design. $\Omega=\left[\alpha, \beta, \gamma_{s}\right]^{\mathrm{T}}$ is called the state variable of the slow loop, and $\omega=\left[\omega_{x 1}, \omega_{y 1}, \omega_{z 1}\right]^{\mathrm{T}}$ is called the state variable of the fast loop. Then, we can use the singular perturbation theory to design a fast-and-slowloop controller for time-scale separation for hypersonic glide vehicle $[17,18]$. The slow loop is designed to produce a slow- loop control output $\omega_{\text {des }}=\left[\omega_{x 1 \text { des }}, \omega_{y 1 \text { des }}, \omega_{z 1 \text { des }}\right]^{\mathrm{T}}$ based on the desired guidance command $\Omega_{\text {des }}=\left[\alpha_{\text {des }}, \beta_{\text {des }}, \gamma_{\text {sdes }}\right]^{\mathrm{T}}$ and is used as the desired value for the fast loop to design the desired command of the control surfaces $\delta=\left[\delta_{a}, \delta_{r}, \delta_{e}\right]^{\mathrm{T}}$. The structure of the fast-and-slow-loop flight control system is shown in Figure 4.

Based on equation (39), by the coupling analysis and time-scale separation principle of the hypersonic glide vehicle, the second innovation of this paper is that dynamic equations of the vehicle can be written into affine nonlinear equations as follows, which can be directly applied to the control law (13) derived from Section 2.3:

$$
\begin{aligned}
& \left\{\begin{array}{l}
\dot{\Omega}=f_{s}(\Omega)+g_{s}(\Omega) \omega, \\
y_{s}=\Omega,
\end{array}\right. \\
& \left\{\begin{array}{l}
\dot{\omega}=f_{f}(\omega)+g_{f}(\omega) \delta, \\
y_{f}=\omega,
\end{array}\right.
\end{aligned}
$$

where $f_{s}(\Omega)=f_{1}(\Omega), g_{s}(\Omega)=N(\Omega), f_{f}(\omega)=f_{2}(\Omega, \omega)$, and $g_{f}(\Omega)=B(\Omega)$ cannot be accurately calculated due to parameter uncertainties. The uncertain parameters include aerodynamic parameters, vehicle body structure parameters, and environment parameters.

The decoupling controller is designed for fast and slow loops by "pitch, yaw, and roll" three-channel design, respectively. There is a one-to-one relationship between the three airflow angles and the control deviation of the three channels, so the feedback controller can be designed first according to the decentralized control theory, and the coupling terms and uncertainties between the channels are not taken into account at this step. Then, the nonlinear extended state observer is designed. The coupling terms and the uncertainties are considered as generalized uncertainty. Its estimated value from the observer is added as a compensation control signal into the decoupling control law, which can eliminate the influence of the coupling terms and the uncertainties. Figure 5 is the decoupling control block diagram of the hypersonic glide vehicle. The deflection angles $\delta_{e}, \delta_{r}$, and $\delta_{a}$ are the inputs of the vehicle's attitude dynamics model, which correspond to $u_{1}, u_{2}$, and $u_{3}$, respectively, in equation (7). Theorem 3 guarantees the convergence of the closed-loop system's tracking error.

\section{Simulation Results}

In order to verify the effectiveness of the proposed method, the mathematical model of the hypersonic glide vehicle is 


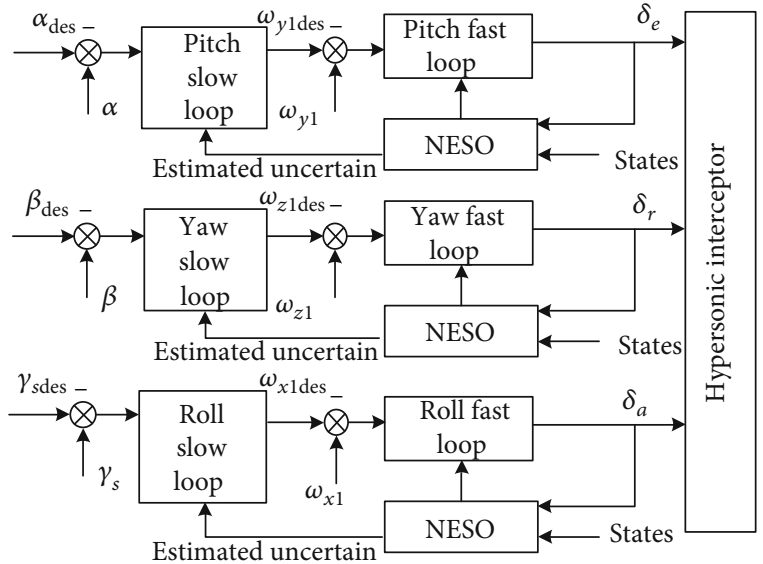

FIGURE 5: Hypersonic glide vehicle's decoupling control block diagram.

built on the MATLAB/Simulink platform, and the designed decoupling controller is simulated and verified.

The main simulation settings and controller parameters are as follows:

(1) The relevant parameters of the aircraft can be referred to the literature [19]

(2) In the simulation, the aerodynamic parameters are offset by $30 \%$ and the moments of inertia are offset by $10 \%$ to verify the robustness of the control method

(3) Considering the characteristics of the hypersonic glide vehicle's flight mode, the following airflow angle command is given: the angle of attack has a step change from 25 degrees to 30 degrees, the bank angle has a step change from 0 degree to 50 degrees, and the sideslip angle remains at 0 degree

(4) The decoupling controller's parameters are set as follows. The subscript " $s$ " represents the slow loop, and the subscript " $f$ " represents the fast loop. Thus, $k_{s}$ represents the controller's feedback gain of the slow loop, and $k_{f}$ represents the controller's feedback gain of the fast loop. The feedback gains of the pitch channel are $k_{s}=3$ and $k_{f}=5$. The feedback gains of the yaw channel are $k_{s}=3$ and $k_{f}=10$. And the feedback gains of the roll channel are $k_{s}=4$ and $k_{f}=7$. The parameters of the NESO are set as $\alpha^{*}=0.7, \delta=0.1$, $\beta_{1}=100$, and $\beta_{2}=300$

(5) The traditional subchannel feedback control method is introduced and compared with the NESO-based decoupling control method designed in this paper, where the feedback control parameters are chosen to be the same values

(6) Figures 6-8 show the performances of the actual airflow angles tracking the commands.

It can be seen from Figures $6-8$ that the tracking performances (overshoot and settling time) of the decoupling

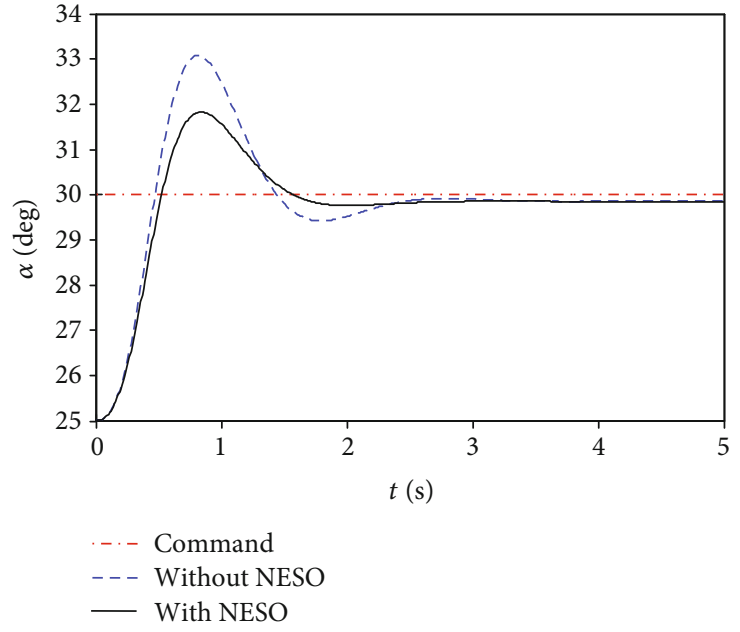

Figure 6: Angle of the attack tracking diagram.

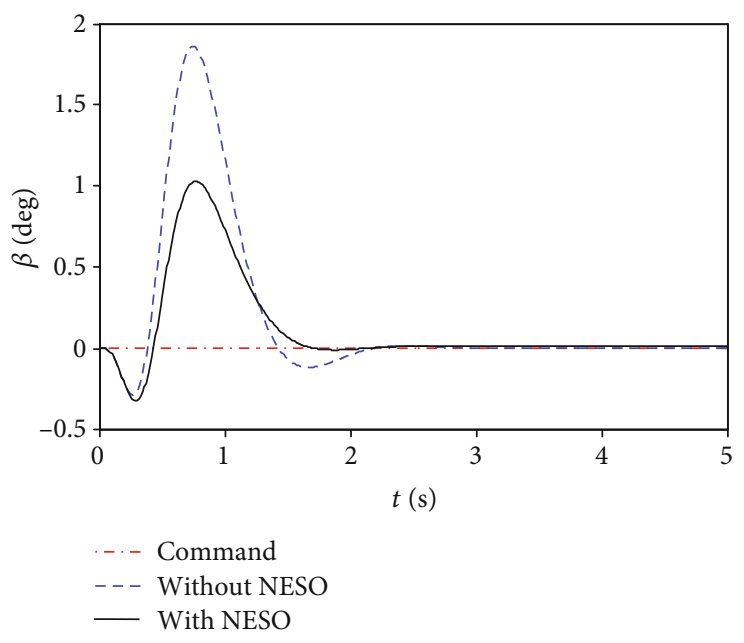

Figure 7: Sideslip angle tracking diagram.

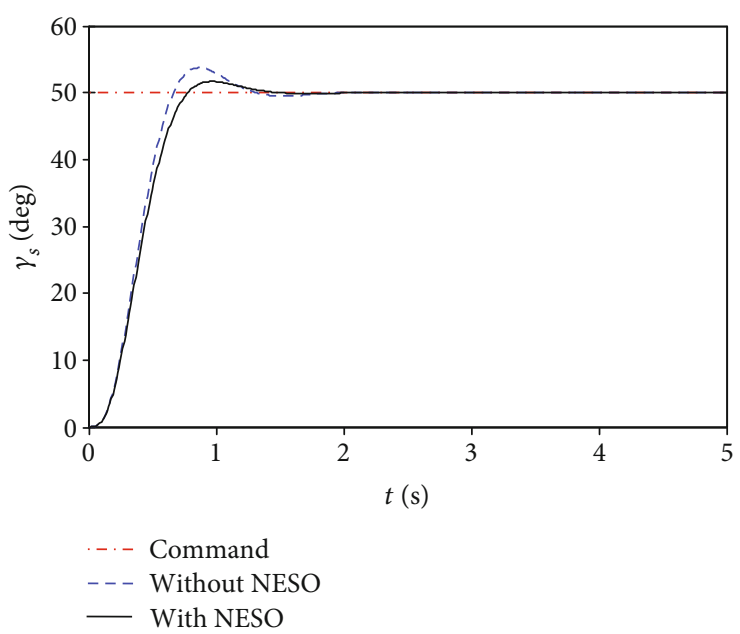

FIgURE 8: Bank angle tracking diagram. 
control based on the NESO are better than those of the traditional subchannel feedback control method. The maximum tracking error is about 2 degrees smaller, and the airflow angle can converge faster to the range of the allowable error. In the yaw channel, the traditional subchannel control method has the relatively large sideslip angle, and the local thermal effect induced by this situation is a serious threat to the safe flight of the hypersonic glide vehicle. The method designed in this paper can limit the sideslip angle to less than 1 degree. For the steady-state error, sometimes the steadystate error of the controller with the NESO will be a little larger than that of the controller without NESO since the highfrequency chattering phenomenon is occurring. The chattering phenomenon is hard to be eliminated completely due to the characteristic of the sliding mode technique used in NESO. Figures 6 and 7 also indicate the phenomenon. However, the overshoot and settling time of the controller with the NESO are both superior to those of the controller without the NESO. The performances of the controller with the NESO are better than those of the controller without the NESO in general.

\section{Conclusions and Future Work}

In this paper, a decoupling control method is proposed for a class of nonlinear MIMO system based on decentralized control theory of Tornambè and NESO. According to the idea of decentralized control, the coupling dynamics and uncertainty are reduced to generalized uncertainties, and then the NESO method is used to give the estimated value which is added as a compensation signal to the decentralized control law. The proposed method is applied to the attitude control problem of a hypersonic glide vehicle. The theoretical derivation and simulation results verify the effectiveness of the proposed method, which is superior to the traditional subchannel control design method.

The future work will focus on how to design a new linear motion controller to form a closed-loop linear motion system; thus, the stability discussion of the linear motion dynamics will be remarked to make the research work more convincing.

\section{Data Availability}

The data used to support the findings of this study are currently under embargo while the research findings are commercialized. Requests for data, 6 months after publication of this article, will be considered by the corresponding author.

\section{Conflicts of Interest}

The authors declare that they have no conflicts of interest to this work.

\section{Acknowledgments}

This work was supported by the National Key R\&D Program of China (Grant Nos. 2017YFD0701003 from 2017YFD 0701000, 2016YFD0200702 from 2016YFD0200700, and 2018 YFD0700603 from 2018YFD0700600); the National
Natural Science Foundation of China (Grant Nos. 51979275); the Jilin Province Key R\&D Plan Project (Grant Nos. 20180201036SF and 20170204008SF); the Open Fund of Synergistic Innovation Center of Jiangsu Modern Agricultural Equipment and Technology, Jiangsu University (Grant No. 4091600015); the Open Research Fund of State Key Laboratory of Information Engineering in Surveying, Mapping and Remote Sensing, Wuhan University (Grant No. 19R06); and the Chinese Universities Scientific Fund (Grant Nos. 10710301, 1071-31051012, and 1071-31051361). And the authors would like to acknowledge the support of Dr. Chen Bai at Ministry of Industry and Information Technology of the People's Republic of China.

\section{References}

[1] R. George, "The common aero vehicle-space delivery system of the future," in AIAA Space Technology Conference \& Exposition, p. 4435, Albuquerque, NM, USA, September 1999.

[2] P. L. Moses, V. L. Rausch, L. T. Nguyen, and J. R. Hill, "NASA hypersonic flight demonstrators-overview, status, and future plans," Acta Astronautica, vol. 55, no. 3-9, pp. 619-630, 2004.

[3] P. Wang, G. J. Tang, and J. Wu, "Sliding mode decoupling control of a generic hypersonic vehicle based on parametric commands," Science China Information Sciences, vol. 58, no. 5, pp. 1-14, 2015.

[4] G. Li, H. Zhang, and G. Tang, "Maneuver characteristics analysis for hypersonic glide vehicles," Aerospace Science and Technology, vol. 43, pp. 321-328, 2015.

[5] F. Q. Zhou, Y. Wang, J. Zhou, and J. G. GUO, "Design of variable structure controller for hypersonic coupled vehicle system," Journal of Astronautics, vol. 32, no. 1, pp. 66-71, 2011.

[6] X. Zeng, "Design of control systems for missiles based on dynamic inversion and decentralized control," Journal of Beijing University of Aeronautics and Astronautics, vol. 33, pp. 1303-1307, 2007.

[7] W. Siwakosit, S. A. Snell, and R. A. Hess, "Robust flight control design with handling qualities constraints using scheduled linear dynamic inversion and loop-shaping," IEEE Transactions on Control Systems Technology, vol. 8, no. 3, pp. 483-494, 2000.

[8] L. C. Fu, W. D. Chang, J. H. Yang, and T. S. Kuo, "Adaptive robust bank-to-turn missile autopilot design using neural networks," Journal of Guidance, Control, and Dynamics, vol. 20, no. 2, pp. 346-354, 2012.

[9] S. Kang, H. J. Kim, J. I. Lee, B. E. Jun, and M. J. Tahk, "Roll-pitch-yaw integrated robust autopilot design for a high angle-of-attack missile," Journal of Guidance, Control, and Dynamics, vol. 32, no. 5, pp. 1622-1628, 2009.

[10] A. Tornambè and P. Valigi, "A PID controller for the robust stabilization of SISO linear systems," Applied Mathematics Letters, vol. 5, no. 4, pp. 15-18, 1992.

[11] A. Tornambè and P. Valigi, "A decentralized controller for the robust stabilization of a class of MIMO linear systems," Systems and Control Letters, vol. 18, no. 5, pp. 383-390, 1992.

[12] A. Tornambè and P. Valigi, "A decentralized controller for the robust stabilization of a class of MIMO dynamical systems," in American Control Conference, pp. 3284-3288, Chicago, IL, USA, June 1992.

[13] A. Tornambè and P. Valigi, "A decentralized controller for the robust stabilization of a class of MIMO dynamical systems," 
Journal of Dynamic System, Measurement, and Control, vol. 116, no. 2, pp. 293-304, 1994.

[14] Y. Huang and J. Han, "Analysis and design for the second order nonlinear continuous extended states observer," Chinese Science Bulletin, vol. 45, no. 21, pp. 1938-1944, 2000.

[15] X. Qu and Z. Ren, "Adaptive robust control based on T-S fuzzy-neural systems for a hypersonic vehicle," in 2011 International Conference on Electrical and Control Engineering, pp. 535-538, Yichang, China, Sept. 2011.

[16] D. Feng and X. Wang, "Modeling and coupling effects analysis of a high-speed aircraft," in IEEE/ASME International Conference on Advanced Intelligent Mechatronics, pp. 1137-1140, Xian, China, July 2008.

[17] T. Bevacqua, E. Best, A. Huizenga, D. Cooper, and J. J. Zhu, "Improved trajectory linearization flight controller for reusable launch vehicles," in 42nd AIAA Aerospace Sciences Meeting and Exhibit, p. 875, 2004.

[18] X. Chen, Z. Hou, and J. Liu, "Nonlinear bank-to-turn autopilot design for hypersonic gliding vehicle," in 3rd International Symposium on Systems and Control in Aeronautics and Astronautics, pp. 1036-1041, Harbin, China, June 2010.

[19] A. D. Rosello, A Vehicle Health Monitoring System for the Space Shuttle Reaction Control System during Reentry, Massachusetts Institute of Technology, Cambridge, 1995. 